\title{
COLCHICINE IS ASSOCIATED WITH LOWER CARDIOVASCULAR MORBIDITY IN GOUT
}

\author{
Claudiu Popescu ${ }^{1,2}$, Warda Abukar ${ }^{1}$, Alexandra Hutan ${ }^{1,3}$, Denisa Predeteanu ${ }^{1,3}$ \\ ${ }^{1}$ Carol Davila University of Medicine and Pharmacy, Bucharest, Romania \\ ${ }^{2}$ Dr. Ion Stoia Clinical Center for Rheumatic Diseases, Bucharest, Romania \\ ${ }^{3}$ Sfanta Maria Clinical Hospital, Bucharest, Romania
}

\begin{abstract}
Objective. This exploratory study aimes at comparing Romanian gout patients with or without colchicine treatment in order to determine if colchicine is associated with a lower cardiovascular morbidity.

Methods. The study was cross-sectionally designed to include all the patients randomly admitted to the hospital between January and July 2015 and discharged with a diagnosis of idiopathic gout according to their attending physicians. The clinical observation sheets served as retrospective sources of information regarding study variables (demographics, addictive behavior, gout phenotype and treatment, cardiovascular morbidity). Data analysis included non-parametric tests and binary logistic regressions (significant if $p<0.05$ ).

Results. The sample included 96 subjects, predominantly men $(77.1 \%)$, with a mean age of 62.7 years. 60 $(62.5 \%)$ subjects received colchicine. Compared to patients who didn't receive colchicine, they had a significantly lower prevalence of ischemic heart disease, chronic heart failure and atherosclerosis $(p<0.05)$. Colchicine was a significant protective factor for atherosclerosis (OR $=0.243 ; 95 \% \mathrm{Cl}: 0.076-0.784)$.

Conclusions. Primary and secondary cardiovascular prevention may benefit from low doses of colchicine. Further prospective blind randomized clinical trials are needed to test these hypotheses.
\end{abstract}

Keywords: colchicine, atherosclerosis, chronic heart failure, gout

\section{INTRODUCTION}

Colchicine is a plant alkaloid used successfully in rheumatology, mainly for the evidence-based treatment and prophylaxis of acute gout (1-4), Behçet's disease (5) and familial Mediterranean fever (6). Due to similar efficacy and better safety, current recommendations are to use low doses of colchicine (1.8 $\mathrm{mg}$ in 1 hour) rather than high doses $(4.8 \mathrm{mg}$ in 6 hours) for the treatment of acute gout $(7,8)$. Colchicine's anti-inflammatory effect in gout indirectly and nonspecifically results from the inhibition of cytoskeleton-dependent leukocyte functions: colchicine binds to tubulins and therefore prevents their polymerization into microtubules (cytoplasmic components of the cytoskeleton), which in turn inhibits cell division (e.g. expansion of activated leukocytes), cell motility (e.g. migration, chemotaxix and adhesion of neutrophils) and intracellular traffic (e.g. phagocytosis, inflammasomes and release of mediators involved in inflammation and joint dam- age, such as chemotaxins, cytokines, enzymes and free radicals) $(9,10)$.

Recent evidence suggests a possible role of colchicine in cardiovascular medicine (11-14), emerging beyond its indication in recurrent pericarditis (15). Since the pathogenesis of atherosclerosis implies an inflammatory process (16), a theoretical benefit of colchicine in this condition was hypothesized and subsequently it was observed in recent clinical trials which reported its efficacy in preventing cardiovascular events $(17,18)$. Reviews and meta-analyses of these trials reported that colchicine significantly reduces the risk of myocardial infarction, that it has no effect on all-cause mortality, but that it reduces cardiovascular mortality in some meta-analytical models (19-22). Further information is needed and ongoing randomized clinical trials are currently evaluating colchicine in atherosclerotic vascular disease (23), acute coronary syndromes $(24,25)$ and prevention of cardiovascular disease

Correspondence address:

Claudiu Popescu MD, Dr. Ion Stoia Clinical Center for Rheumatic Diseases, 5th Thomas Masaryk Street, district 2, Bucharest

E-mail: claudiu.popescu@reumatologiedrstoia.ro 
$(26,27)$. Such protective cardiovascular effect would greatly benefit gout management since gout itself is associated with increased cardiovascular risk and mortality $(28,29)$. In addition, Romanians are known to have a higher cardiovascular risk than other Europeans (30), therefore it is relevant to investigate the potential cardiovascular benefit of colchicine in this gout population. Thus, this exploratory study aims at comparing Romanian gout patients with or without colchicine treatment in order to determine if colchicine is associated with lower cardiovascular morbidity.

\section{METHODS}

\section{Patients}

Using a cross-sectional design, the study included all the patients randomly admitted between January and July 2015 to the "Sfanta Maria" Clinical Hospital Department of Rheumatology and discharged with a diagnosis of idiopathic gout according to their attending physicians. The cases were identified using the specific M10.0 International Classification of Diseases codes recorded in the digital data records of the hospital. On admission, all patients gave a written informed consent regarding the scientific use of clinical data recorded in the observation sheets. The protocol was approved by the local ethics committee.

\section{Variables}

The clinical medical records served as retrospective sources of information regarding age, gender and residence. Smoking status and alcohol consumption, as declared by the patient, were collected from the clinical interview recorded in writing in each medical record. The clinical examination carried out by each physician and recorded in the medical record allowed the collection of information regarding gout phenotype: the reason for the medical visit (gout attack or follow-up), the number of gout attacks (single or multiple) and the presence of tophi. The local laboratory determined the level of serum uric acid using the same commercially available kits and patients were classified with hyperuricemia if this level was above $7.2 \mathrm{mg} / \mathrm{dL}$ for men or $6.1 \mathrm{mg} / \mathrm{dL}$ for women. Patients were classified with different diagnoses if the medical records contained these diagnoses or if the patient fulfilled specific criteria: arterial hypertension (treatment with anti-hyperten- sive drugs or two blood pressure measurements recorded a systolic pressure $\geq 140 \mathrm{mmHg}$ or a diastolic pressure $\geq 90 \mathrm{mmHg}$ ) (31); ischemic heart disease (specific electrocardiographic signs or history of acute coronary syndromes, stable angina, conduction and rhythm disturbances and ischemic heart failure) (32); congestive heart failure (recorded ejection fraction of $40 \%$ or bellow) (33); atherosclerosis (recorded proof of plaque deposition obtained by carotid ultrasound, coronary magnetic resonance or computed tomography imaging, ankle-brachial index or arterial wave forms) (34); type 2 diabetes mellitus (treatment with insulin or oral hypoglycemic drugs or recorded fasting plasma glucose level above $126 \mathrm{mg} / \mathrm{dL}$ on two different measurements or above $200 \mathrm{mg} / \mathrm{dL}$ in a single measurement) (35); obesity (recorded/calculated body mass index -2 weight divided by square height - above $30 \mathrm{~kg} / \mathrm{m}^{2}$ ); chronic kidney disease (recorded proof of altered kidney function for more than 3 months, namely glomerular filtration rate below $60 \mathrm{~mL} / \mathrm{min} / 1.73 \mathrm{~m}^{2}$, albuminuria, urine sediment abnormalities, electrolyte abnormalities due to tubular disorders, biopsy abnormalities, imaging structural abnormalities and history of kidney transplantation) (36). Prescriptions contained by the medical records allowed the collection of treatment variables: diuretics and other hyperuricemia-inducing drugs, colchicine and uratelowering therapy (Table 1).

\section{Statistics}

Data distribution normality was assessed using descriptive statistics, normality plots, stem-and-leaf plots and the Lillefors corrected KolmogorovSmirnov tests. Age exhibited a normal distribution in the whole sample and was reported as "mean (standard deviation)". Nominal variables were expressed as "absolute value (percentage of group)", their associations with other nominal data were studied using cross-tabs with $\chi^{2}$ tests (or Fisher's exact test) and their age differences were assessed using the non-parametric Mann-Whitney U test. To assess the independent predictive capacity of gout variables for cardiovascular outcomes, binary logistic regression models were created (nominal variables were coded " 0 " for "no" and " 1 " for "yes" if not otherwise stated): firstly, an exploratory forward conditional regression model was created to predict atherosclerosis and it included cardiovascular variables (age, gender, coded " 0 " for "woman" and "1" for "man", 
TABLE 1. General characteristics of the studied sample $(n=96)$

\begin{tabular}{|c|c|c|}
\hline demographics & & \\
\hline males (n) & $74(77.1 \%)$ & \multirow{23}{*}{$\begin{array}{l}\text { Notes: } \\
\text { - age, a normally distributed scale variable, is } \\
\text { reported as "mean (standard deviation)"; nominal } \\
\text { data are reported as "absolute value (percent of } \\
\text { sample size)". } \\
\text { - clinical diagnoses (AHT, type } 2 \text { DM, obesity, IHD, } \\
\text { atherosclerosis, CHF, CKD) are reported according } \\
\text { to the opinion of attending physicians as it } \\
\text { appears in the medical files of each patient } \\
\text { - diuretics refer to: hydrochlorothiazide, } \\
\text { chlorthalidone, triamterene, spironolactone, } \\
\text { amiloride, bumetanide, furosemide, } \\
\text { torsemide, methyclothiazide, acetazolamide, } \\
\text { bendroflumethiazide, indapamide, metolazone, } \\
\text { ethacrynate. } \\
\text { - HU-inducing drugs refer to: cytotoxic drugs, } \\
\text { pyrazinamide, ribavirin, interferon, didanosine, } \\
\text { isotreonin, teophylline, niacin. } \\
\text { - ULT refers to: allopurinol, febuxostat, uricases, } \\
\text { probenecid, sulfinpyrazone, benzbromarone. } \\
\text { Abbreviations: AHT - arterial hypertension; } \\
\text { CHF - congestive heart failure; CKD - chronic } \\
\text { kidney disease; HU - hyperuricemia; } \\
\text { IHD - ischemic heart disease; } n \text { - number; } \\
\text { T2DM - type } 2 \text { diabetes mellitus; ULT - urate } \\
\text { lowering therapy. }\end{array}$} \\
\hline urban dwellers (n) & $66(68.8 \%)$ & \\
\hline age (years) & $62.7(10.8)$ & \\
\hline smokers (n) & $11(11.5 \%)$ & \\
\hline alcohol (n) & $36(37.5 \%)$ & \\
\hline gout phenotype & & \\
\hline acute gout (n) & 50 (52.1\%) & \\
\hline multiple attacks (n) & 70 (72.9\%) & \\
\hline tophi (n) & $20(20.8 \%)$ & \\
\hline $\mathrm{HU}(\mathrm{n})$ & $59(61.5 \%)$ & \\
\hline comorbid & & \\
\hline $\mathrm{AHT}(\mathrm{n})$ & $66(68.8 \%)$ & \\
\hline T2DM (n) & $14(14.6 \%)$ & \\
\hline obesity (n) & $41(42.7 \%)$ & \\
\hline IHD (n) & $41(42.7 \%)$ & \\
\hline atherosclerosis (n) & $27(28.1 \%)$ & \\
\hline $\mathrm{CHF}(\mathrm{n})$ & $21(21.9 \%)$ & \\
\hline CKD (n) & $22(22.9 \%)$ & \\
\hline medication & & \\
\hline diuretics (n) & $27(28.1 \%)$ & \\
\hline other HU-inducers (n) & $4(4.2 \%)$ & \\
\hline colchicine $(n)$ & $60(62.5 \%)$ & \\
\hline $\operatorname{ULT}(n)$ & $84(87.5 \%)$ & \\
\hline
\end{tabular}

smoking, alcohol consumption, diabetes mellitus, obesity, arterial hypertension, ischemic heart disease) and gout variables (gout attack frequency, coded " 0 " for "single attack" and " 1 " for "multiple attacks", tophi, hyperuricemia, urate-lowering therapy, colchicine); secondly, a simultaneous regression model was created to predict atherosclerosis using the significant independent variables identified in the previous step (age, arterial hypertension, obesity and colchicine; Fig. 2). All statistical tests were carried out using IBM SPSS v.20 (IBM Inc., Armonk, N.Y., 2010) for Windows and were considered significant if $p<0.05$. The figures were created using GraphPad Prism 6.0 (GraphPad Software, La Jolla California, USA).

\section{RESULTS}

\section{General characteristics}

The sample included 96 subjects, predominantly males $(77.1 \%)$, with a mean age of 62.7 (10.8) years (Table 1). Approximately one fifth of the subjects had tophaceous gout and half of the subjects had acute gout at the moment of inclusion. Regarding medication, 60 (62.5\%) subjects received colchicine.

\section{Colchicine treatment}

Compared to patients who were not taking colchicine, those with colchicine treatment displayed four significant differences: they had a significantly higher prevalence of acute gout and a significantly lower prevalence of ischemic heart disease, chronic heart failure and atherosclerosis (Table 2, Fig. 1). Binary logistic regression models which included colchicine produced significant results (Fig. 2): while age, obesity and arterial hypertension were as expected significant risk factors for atherosclerosis, colchicine instead was a significant protective factor for atherosclerosis. In the binary logistic regression models created to predict either ischemic heart disease or chronic heart failure, colchicine was not a significant predictor (data not shown).

\section{DISCUSSION}

This pilot study aimed at determining if colchicine is associated with lower cardiovascular morbidity in Romanian gout patients. According to our observations, gout patients on colchicine had a lower prevalence of ischemic heart disease, chronic heart failure and atherosclerosis compared to gout patients 
TABLE 2. Differences between colchicine subgroups

\begin{tabular}{|c|c|c|c|c|}
\hline & \multicolumn{2}{|c|}{ colchicine } & \multirow[b]{2}{*}{$p$} & \multirow{21}{*}{$\begin{array}{l}\text { Notes: } \\
\text { - age is reported as "median } \\
\text { (interquartile range)"; nominal } \\
\text { variables are reported as "percentage } \\
\text { of subgroup (observed absolute } \\
\text { frequency)"; } \\
\text { - p values represent the significance } \\
\text { of the Mann Whitney tests (for } \\
\text { age) and for of } \chi^{2} \text { tests or Fisher's } \\
\text { exact test (for nominal variables), } \\
\text { significant if > } 0.05 \text {. } \\
\text { Abbreviations: AHT - arterial } \\
\text { hypertension; CHF - congestive heart } \\
\text { failure; CKD - chronic kidney disease; } \\
\text { HU - hyperuricemia; IHD - ischemic } \\
\text { heart disease; } n \text { - number; T2DM - } \\
\text { type } 2 \text { diabetes mellitus; ULT - urate } \\
\text { lowering therapy. }\end{array}$} \\
\hline & no $(n=36)$ & yes $(n=60)$ & & \\
\hline males $(n)$ & $75.0 \%(27)$ & $78.3 \%(47)$ & 0.707 & \\
\hline urban dwellers (n) & $69.4 \%(25)$ & $68.3 \%(41)$ & 0.909 & \\
\hline age (years) & $65(18)$ & $61(13)$ & 0.126 & \\
\hline smokers (n) & $8.3 \%(3)$ & $13.3 \%(8)$ & 0.528 & \\
\hline alcohol (n) & $33.3 \%(12)$ & $40.0 \%(24)$ & 0.514 & \\
\hline acute gout (n) & $36.1 \%(13)$ & $61.7 \%(37)$ & 0.015 & \\
\hline multiple attacks (n) & $80.6 \%(29)$ & $68.3 \%(41)$ & 0.192 & \\
\hline tophi (n) & $22.2 \%(8)$ & $20.0 \%(12)$ & 0.795 & \\
\hline $\mathrm{HU}(\mathrm{n})$ & $52.8 \%(19)$ & $66.7 \%(40)$ & 0.176 & \\
\hline $\mathrm{AHT}(\mathrm{n})$ & $72.2 \%(26)$ & $66.7 \%(40)$ & 0.570 & \\
\hline T2DM (n) & $13.9 \%(5)$ & $15.0 \%(9)$ & 0.881 & \\
\hline obesity (n) & $41.7 \%(15)$ & $43.3 \%(26)$ & 0.873 & \\
\hline$I H D(n)$ & $55.6 \%(20)$ & $35.0 \%(21)$ & 0.049 & \\
\hline atherosclerosis ( $n$ ) & $44.4 \%(16)$ & $18.3 \%(11)$ & 0.006 & \\
\hline $\mathrm{CHF}(n)$ & $33.3 \%(12)$ & $15.0 \%(9)$ & 0.035 & \\
\hline CKD (n) & $30.6 \%(11)$ & $18.3 \%(11)$ & 0.168 & \\
\hline diuretics $(n)$ & $36.1 \%(13)$ & $23.3 \%(14)$ & 0.178 & \\
\hline HU-inducers (n) & $27.8 \%(10)$ & $21.7 \%(13)$ & 0.497 & \\
\hline ULT (n) & $91.7 \%(33)$ & $85.0 \%(51)$ & 0.526 & \\
\hline
\end{tabular}
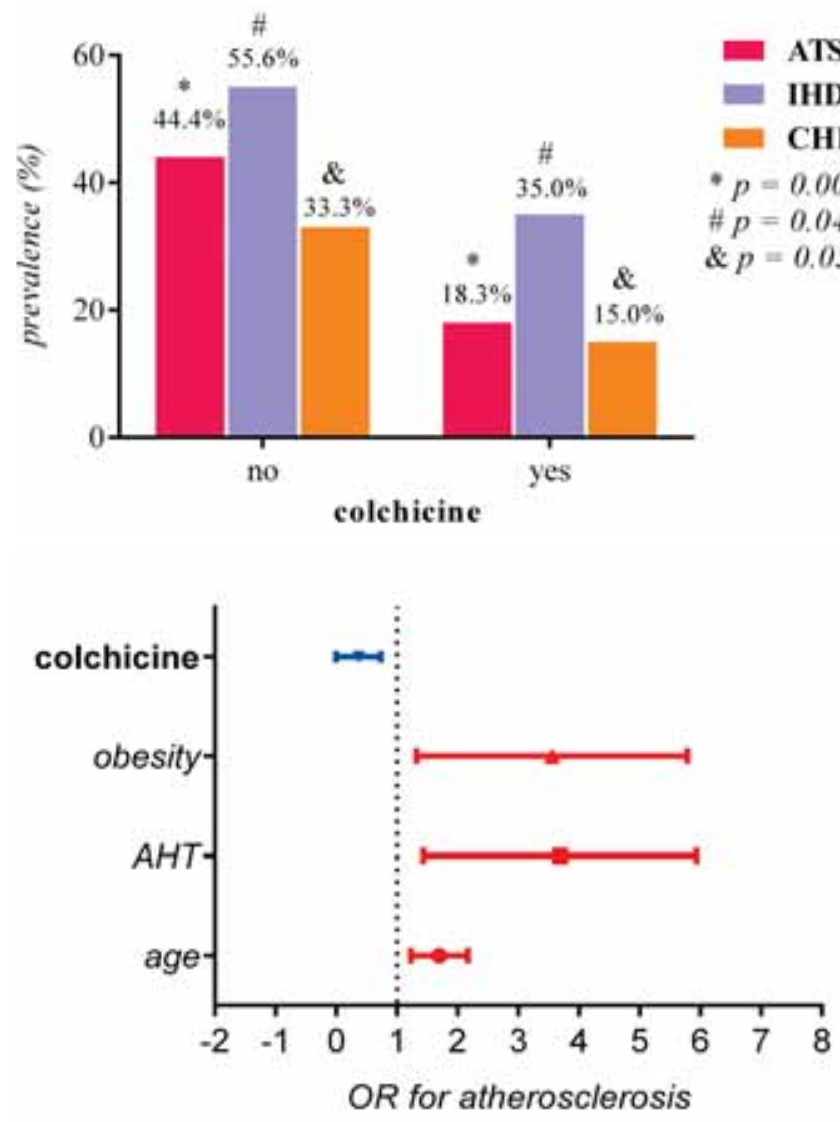

without colchicine treatment (Table 2, Fig. 1), and colchicine was a significant protective factor for atherosclerosis (Fig. 2).

To our knowledge, there are no other studies which report such results explicitly. The literature
FIGURE 1. The prevalence of atherosclerosis (ATS), ischemic heart disease (IHD) and chronic heart failure (CHF) among patients treated or not with colchicine. Notes: $p$ values represent the significance of $\chi^{2}$ tests; the percentages represent the proportion of subjects relative to colchicine subgroups

FIGURE 2. Significant predictors in the standard logistic regression model used to predict atherosclerosis. Notes: age is a scale variable, while obesity, arterial hypertension (AHT) and colchicine are nominal variables, coded "0" for "no" and "1" for "yes"; the model was significant: $X^{2}=39.9, p<0.001$; the model increased the predictive capacity from $71.9 \%$ (observed) to $80.2 \%$ (predicted) and it explained half of atherosclerosis variability (Nagelkerke $R^{2}=0.489$ ); odds ratios (OR) with $95 \%$ confidence intervals: $O R_{\text {age }}=1.696(1.229-2.169)$, $O R_{A H T}=3.698(1.429-5.927), O R_{\text {obesity }}=3.146(1.561$ $5.965), O R_{\text {colchicine }}=0.243(0.076-0.784)$

does contain similar reports which are adjacently concordant with our observations and which focus mainly on acute coronary syndromes and cardiovascular death. For example, in a retrospective, crosssectional study on 1,288 gout patients, Crittenden et 
al. (37) found that patients on colchicine had a significantly lower prevalence of myocardial infarction compared with patients without colchicine (1.2\% versus $2.6 \% ; \mathrm{p}=0.03$ ). Solomon et al. (38) reported that colchicine reduces the risk of cardiovascular events by $49 \%$ and the risk of all-cause mortality by $73 \%$ in gout patients compared to controls, although with some methodological and confounding issues (39-41). Nidorf et al. (17) designed a prospective, randomized, observer-blinded study which followed, for 3 years, 532 patients with stable coronary disease randomly assigned to receive a low dose of colchicine or no colchicine, reporting that the primary outcome (composite incidence of acute coronary syndrome, out-of-hospital cardiac arrest or non-cardiometabolic ischemic stroke) occurred significantly less frequent in the colchicine subgroup $(5.3 \%$ compared to $16.0 \% ; \mathrm{p}<0.001)$. Recent meta-analyses of studies done mostly in the general population tend to confirm the benefits of colchicine in cardiovascular medicine: Verma et al. (22) reported that colchicine reduced composite cardiovascular outcomes by $60 \%$ in patients with established cardiovascular disease, while Hemkens et al. $(19,20)$ reported that colchicine reduced the risk for fatal and non-fatal myocardial infarction, but it had no effect on all-cause mortality, heart failure and stroke.

In this context, if colchicine has indeed a significant cardioprotective effect, our observations that gout patients on colchicine had fewer cardiovascular comorbidities should model the clinical reality. Because of the retrospective study design, we cannot certainly state that our patients had fewer cardiovascular comorbidities because of colchicine, we can only confirm the association of colchicine treatment with better cardiovascular profiles. To get closer to causal inference, we would need a prospective trial which would record treatment duration in time (its lack in our study being an important limitation), dose-effect relationship and which would randomly assign patients to colchicine treatment in order to eliminate potential selection bias done by attending physicians in clinical practice (doctors might prescribe colchicine to patients who already have a better cardiovascular profile). Another limitation of this study is the fact that it only observed gout in-patients (a subpopulation which very probably has an increased cardiovascular morbidity due to the rheumatic diagnosis and to the need for hospitalization), without a matched control group.
The mechanisms by which colchicine would cause a lower prevalence of atherosclerosis and chronic heart failure are intriguing. Since literature focused on acute coronary syndromes, the fundamental research of the cardiovascular effect of colchicine focused on atherosclerotic plaque instability. Because neutrophils are present and are active in the atherosclerotic plaque during its entire existence (42), the ability of colchicine to impair neutrophil function seems to be the most likely mechanism of action responsible for the risk reduction of acute cardiovascular events (18). Moreover, colchicine seems to inhibit platelet aggregation and stabilize the vascular endothelium (11), further reducing the risk of plaque rupture. Our colchicine-treated patients had a lower prevalence of atherosclerosis, suggesting that colchicine does more than stabilize atherosclerotic plaques (secondary cardiovascular prevention): it might even prevent their formation (primary cardiovascular prevention) - a hypothesis which was demonstrated in animals (18) and which is currently being studied in humans. Since colchicine has a nonspecific anti-inflammatory mode of action, it might disrupt plaque formation from the very beginning, inhibiting stimulated endothelial cells to express adhesion molecules and pro-inflammatory cytokines - processes which depend on proper cytoskeleton function. As the pathogenesis of atherosclerosis progresses, colchicine can inhibit the cellular function of non-neutrophil cells involved (macrophages, T lymphocytes and vascular fibroblasts).

Regarding colchicine and chronic heart failure, the literature is conflicting. Cicogna et al. $(43,44)$ first reported that colchicine fails to improve cardiac muscle function in rats with heart failure, but Saji et al. (45) observed that colchicine inhibits cardiomyocyte apoptosis (an important pathogenic mechanism in heart failure). Despite this evidence from fundamental science research, the plausibility of colchicine efficacy in heart failure still remained. In order to address the clinical significance of colchicine in heart failure, Deftereos et al. (46) designed a randomized placebo-controlled trial which sought improvement in New York Heart Association (NYHA) class of colchicine-treated patients with heart failure: colchicine was effective in lowering C-reactive protein and interleukin- 6 levels, but the actively treated patients did not improve their NYHA grades. From the animal studies and from this randomized trial it seems clear that colchicine is not indicated for the treatment of heart failure. Our observation of a 
lower prevalence of heart failure among patients taking colchicine raises the question whether this drug can prevent heart failure, maybe by reducing intracardiac atherosclerosis, a case in which a prospective cohort study would be useful. Since the mechanism of action is not specific enough and since there are significant issues regarding gastrointestinal tolerance, colchicine may never find its way in the treatment schemes of heart failure.

\section{CONCLUSION}

Colchicine might be a significant protective factor for atherosclerosis and it might be associated with lower prevalence of ischemic heart disease and chronic heart failure. Consequently, primary and secondary cardiovascular prevention may benefit from low doses of colchicine. Further prospective blind randomized clinical trials are needed to test these hypotheses.

\section{Acknowledgment}

The authors gratefully acknowledge the physicians from the Department of Internal Medicine and Rheumatology of "Sfanta Maria" Clinical Hospital for facilitating data collection.

13. Imazio M., Gaita F. Colchicine for cardiovascular medicine. Future cardiology 2016, 12(1):9-16.

14. Imazio M., Gaita F. Is colchicine a promising novel treatment option for cardiovascular medicine? Heart 2016, 102(8):569-570.

15. Raval J., Nagaraja V., Eslick G.D., Denniss A.R. The Role of Colchicine in Pericarditis--A Systematic Review and Meta-analysis of Randomised Trials. Heart, lung \& circulation 2015, 24(7):660-666

16. Pant S., Deshmukh A., Gurumurthy G.S., Pothineni N.V., Watts T.E., Romeo F., Mehta J.L. Inflammation and atherosclerosis revisited. Journal of cardiovascular pharmacology and therapeutics 2014, 19(2):170-178.

17. Nidorf S.M., Eikelboom J.W., Budgeon C.A., Thompson P.L. Low-dose colchicine for secondary prevention of cardiovascular disease. Journal of the American College of Cardiology 2013, 61(4):404-410.

18. Nidorf S.M., Eikelboom J.W., Thompson P.L. Colchicine for secondary prevention of cardiovascular disease. Current atherosclerosis reports 2014, 16(3):391.

19. Hemkens L.G., Ewald H., Gloy V.L., Arpagaus A., Olu K.K., Nidorf M., Glinz D., Nordmann A.J., Briel M. Colchicine for prevention of cardiovascular events. The Cochrane database of systematic reviews 2016, 1:CD011047.

20. Hemkens L.G., Ewald H., Gloy V.L., Arpagaus A., Olu K.K., Nidorf M., Glinz D., Nordmann A.J., Briel M. Cardiovascular effects and safety of long-term colchicine treatment: Cochrane review and meta-analysis. Heart 2016, 102(8):590-596.

21. Tong D.C., Wilson A.M., Layland J. Colchicine in cardiovascular disease: an ancient drug with modern tricks. Heart 2016.

22. Verma S., Eikelboom J.W., Nidorf S.M., Al-Omran M., Gupta N., Teoh H., Friedrich J.O. Colchicine in cardiac disease: a systematic review and meta-analysis of randomized controlled trials. BMC Cardiovasc Disord 2015, 15:96.

23. A Randomized, Double-blind, Placebo-controlled Study to Evaluate the Effects of Colchicine on Vascular Inflammation as Assessed With Position Emission Tomography (PET) Imaging in Patients With Atherosclerotic Vascular Disease (COLPET). ClinicalTrialsgov Bethesda (MD): National Library of Medicine (US), 2000-2016(https://clinicaltrials.gov/ct2/show/NCT02162303).

24. Colchicine for Acute Coronary Syndromes. A Multicenter Double Blind Randomized Trial. ClinicalTrialsgov Bethesda (MD): National Library of Medicine (US), 2000-2016(https://clinicaltrials.gov/ct2/ show/study/NCT01906749).

25. Colchicine Compared With Placebo to Reduce Hs-CRP in Patients With Acute Coronary Syndromes- Targeting Inflammation in Atherosclerosis Trial. ClinicalTrialsgov Bethesda (MD): National Library of Medicine (US), 2000-2016(https://clinicaltrials.gov/ct2/ show/NCT00754819). 
26. Anti-platelet Effects of Colchicine in Healthy Volunteers. ClinicalTrialsgov Bethesda (MD): National Library of Medicine (US), 2000-2016 (https://clinicaltrials.gov/ct2/show/study/NCT02140372)

27. The LoDoCo2 Trial: A randomised controlled trial on the effect of low dose Colchicine for secondary prevention of cardiovascular disease in patients with established, stable coronary artery disease. Australian New Zeeland Clinical Trials Registry, 2016 (https://www.anzctr.org.au/Trial/Registration/TrialReview. aspx?ACTRN=12614000093684).

28. Puig J.G., Martinez M.A. Hyperuricemia, gout and the metabolic syndrome. Curr Opin Rheumatol 2008, 20(2):187-191.

29. Kuo C.F., See L.C., Luo S.F., Ko Y.S., Lin Y.S., Hwang J.S., Lin C.M., Chen H.W., Yu K.H. Gout: an independent risk factor for allcause and cardiovascular mortality. Rheumatology (Oxford) 2010, 49(1):141-146.

30. Dorobantu M., Badila E., Ghiorghe S., Darabont R.O., Olteanu M., Flondor P. Total cardiovascular risk estimation in Romania. Data from the SEPHAR study. Romanian journal of internal medicine $=$ Revue roumaine de medecine interne 2008 , 46(1):29-37.

31. Mancia G., Fagard R., Narkiewicz K., Redon J., Zanchetti A., Bohm M., Christiaens T., Cifkova R., De Backer G., Dominiczak A. et al. $2013 \mathrm{ESH} / \mathrm{ESC}$ guidelines for the management of arterial hypertension: the Task Force for the Management of Arterial Hypertension of the European Society of Hypertension (ESH) and of the European Society of Cardiology (ESC). European heart journal 2013, 34(28):2159-2219.

32. Fihn S.D., Gardin J.M., Abrams J., Berra K., Blankenship J.C., Dallas A.P., Douglas P.S., Foody J.M., Gerber T.C., Hinderliter A.L. et al. 2012 ACCF/AHA/ACP/AATS/PCNA/SCAI/STS guideline for the diagnosis and management of patients with stable ischemic heart disease: a report of the American College of Cardiology Foundation/American Heart Association task force on practice guidelines, and the American College of Physicians, American Association for Thoracic Surgery, Preventive Cardiovascular Nurses Association, Society for Cardiovascular Angiography and Interventions, and Society of Thoracic Surgeons. Circulation 2012, 126(25):e354-471.

33. Writing Committee M., Yancy C.W., Jessup M., Bozkurt B., Butler J., Casey D.E., Jr., Drazner M.H., Fonarow G.C., Geraci S.A., Horwich T. et al. 2013 ACCF/AHA guideline for the management of heart failure: a report of the American College of Cardiology Foundation/American Heart Association Task Force on practice guidelines. Circulation 2013, 128(16):e240-327.

34. Bild D.E., Bluemke D.A., Burke G.L., Detrano R., Diez Roux A.V., Folsom A.R., Greenland P., Jacob D.R., Jr., Kronmal R., Liu K. et al. Multi-Ethnic Study of Atherosclerosis: objectives and design. American journal of epidemiology 2002, 156(9):871-881.
35. American Diabetes A: Diagnosis and classification of diabetes mellitus. Diabetes care 2014, 37 Suppl 1:S81-90.

36. Group KDIGOKCW: KDIGO 2012 clinical practice guideline for the evaluation and management of chronic kidney disease. Kidney International Supplements 2013, 3(1):1-150.

37. Crittenden D.B., Lehmann R.A., Schneck L., Keenan R.T., Shah B., Greenberg J.D., Cronstein B.N., Sedlis S.P., Pillinger M.H. Colchicine use is associated with decreased prevalence of myocardial infarction in patients with gout. The Journal of rheumatology 2012, 39(7):1458-1464.

38. Solomon D.H., Liu C.C., Kuo I.H., Zak A., Kim S.C. Effects of colchicine on risk of cardiovascular events and mortality among patients with gout: a cohort study using electronic medical records linked with Medicare claims. Annals of the rheumatic diseases 2015.

39. Giannopoulos G., Deftereos S. Effects of colchicine on risk of cardiovascular events among patients with gout: as evidence accrues, is it time for a randomised trial? Annals of the rheumatic diseases 2016.

40. Nielen J.T., Oshagbemi O., de Vries F., Burden A.M. Effects of colchicine on risk of cardiovascular events and mortality among patients with gout: the effect of index date selection? Annals of the rheumatic diseases 2016.

41. Solomon D.H., Kim S.C. Potential confounding in colchicine and cardiovascular disease study. Annals of the rheumatic diseases 2016.

42. Carbone F., Mach F., Montecucco F. Update on the role of neutrophils in atherosclerotic plaque vulnerability. Current drug targets 2015, 16(4):321-333.

43. Cicogna A.C., Brooks W.W., Hayes J.A., Robinson K.G., Sen S., Conrad C.H., Bing O.H. Effect of chronic colchicine administration on the myocardium of the aging spontaneously hypertensive rat. Molecular and cellular biochemistry 1997, 166(1-2):45-54.

44. Cicogna A.C., Robinson K.G., Conrad C.H., Singh K., Squire R., Okoshi M.P., Bing O.H. Direct effects of colchicine on myocardial function: studies in hypertrophied and failing spontaneously hypertensive rats. Hypertension 1999, 33(1):60-65.

45. Saji K., Fukumoto Y., Suzuki J., Fukui S., Nawata J., Shimokawa H. Colchicine, a microtubule depolymerizing agent, inhibits myocardial apoptosis in rats. The Tohoku journal of experimental medicine 2007, 213(2):139-148.

46. Deftereos S., Giannopoulos G., Panagopoulou V., Bouras G., Raisakis K., Kossyvakis C., Karageorgiou S., Papadimitriou C., Vastaki M., Kaoukis A. et al. Anti-inflammatory treatment with colchicine in stable chronic heart failure: a prospective, randomized study. JACC Heart failure 2014, 2(2):131-137. 